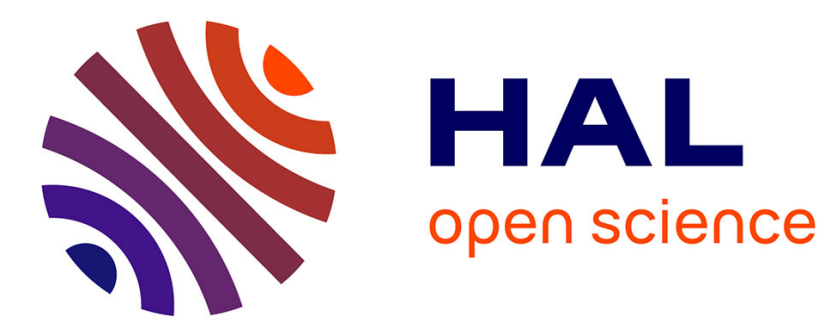

\title{
Time-efficient sparse analysis of histopathological Whole Slide Images
}

\author{
Chao-Hui Huang, Antoine Veillard, Nicolas Lomenie, Daniel Racoceanu, \\ Ludovic Roux
}

\section{- To cite this version:}

Chao-Hui Huang, Antoine Veillard, Nicolas Lomenie, Daniel Racoceanu, Ludovic Roux. Time-efficient sparse analysis of histopathological Whole Slide Images. Computerized Medical Imaging and Graphics, 2010, pp.5. hal-00553877

\section{HAL Id: hal-00553877 \\ https://hal.science/hal-00553877}

Submitted on 10 Jan 2011

HAL is a multi-disciplinary open access archive for the deposit and dissemination of scientific research documents, whether they are published or not. The documents may come from teaching and research institutions in France or abroad, or from public or private research centers.
L'archive ouverte pluridisciplinaire HAL, est destinée au dépôt et à la diffusion de documents scientifiques de niveau recherche, publiés ou non, émanant des établissements d'enseignement et de recherche français ou étrangers, des laboratoires publics ou privés. 


\title{
Time-efficient sparse analysis of histopathological Whole Slide Images
}

\author{
Chao-Hui Huang ${ }^{\mathrm{b}, \mathrm{c}}$, Antoine Veillard ${ }^{\mathrm{b}, \mathrm{c}}$, Nicolas Loménie ${ }^{\mathrm{a}, \mathrm{b}}$, Daniel Racoceanu ${ }^{\mathrm{a}, \mathrm{b}, \mathrm{c}, \mathrm{d}}$, Ludovic Roux ${ }^{\mathrm{b}, \mathrm{e}, *}$ \\ ${ }^{a}$ Centre National de la Recherche Scientifique (CNRS), Paris, France \\ ${ }^{b} I P A L$ (Image 83 Pervasive Access Lab), International Mixed Research Unit UMI CNRS 2955 (CNRS, NUS, I2R/A*STAR, UJF), \\ Singapore (http://ipal.i2r.a-star.edu.sg/) \\ ${ }^{c}$ National University of Singapore, Singapore \\ ${ }^{d}$ University of Franche-Comté, Besançon, France \\ ${ }^{e}$ University Joseph Fourier, Grenoble, France
}

\begin{abstract}
Histopathological examination is a powerful method for the prognosis of critical diseases. But, despite significant advances in high-speed and high-resolution scanning devices or in virtual exploration capabilities, the clinical analysis of Whole Slide Images (WSI) largely remains the work of human experts. We propose an innovative platform in which multi-scale computer vision algorithms perform fast analysis of a histopathological WSI. It relies on specific high and generic low resolution image analysis algorithms embedded in a multi-scale framework to rapidly identify the high power fields of interest used by the pathologist to assess a global grading. GPU technologies as well speed up the global time-efficiency of the system. In a sense, sparse coding and sampling is the keystone of our approach. In terms of validation, we are designing a computer-aided breast biopsy analysis application based on histopathology images and designed in collaboration with a pathology department. The current ground truth slides correspond to about 36,000 high magnification (40X) high power fields. The time processing to achieve automatic WSI analysis is on a par with the pathologist's performance (about ten minutes a WSI), which constitutes by itself a major contribution of the proposed methodology.
\end{abstract}

Keywords: Histopathology, breast cancer, Whole Slide Image, multi-scale analysis, dynamic sampling, virtual microscope, Graphics Processing Unit

\section{Introduction}

Histopathology is widely accepted as a powerful gold standard for prognosis in critical diseases such as breast, prostate, kidney and lung cancers, allowing to narrow borderline diagnosis issued from standard macroscopic noninvasive analysis such as mammography and ultrasonography. At the molecular/genetic scale as well challenging methods recently emerged for clinical diagnosis purposes. However, histomorphology as operated in hospitals is and will remain the basis for most cancer classification.

The histopathological image analysis process has largely remained the work of human experts so far. At the hospital level, the task consists in the daily examination of hundreds of slides, directly impacting critical diagnosis and treatment decisions. According to pathologists' opinion [1], such a tedious manual work is often inconsistent and subjective, lacking traceability and computer assisted analysis/annotation/grading support tools. In addition, hospitals will have to manage a shortage of expert pathologists keen at doing this kind of unrewarding tasks.

A few image analysis algorithms and automated grading systems dedicated to breast histopathology images have

\footnotetext{
* Corresponding author

Email address: vislr@i2r.a-star.edu.sg (Ludovic Roux)
}

already been studied. Estévez et al. [2] and Schnorrenber et al. [3] worked on Fine Needle Aspiration (FNA) biopsies. FNA images are relatively easier to analyze than WSIs since such an examination has limited diagnostic options and produces mostly well separated cells over a wellcontrasted background. Petushi et al. $[4,5]$ introduced a system able to label several histological and cytological microstructures in high resolution frames, including different grades of epithelial cells, fat cells and stroma. Doyle et al. $[6,7]$ proposed a method based on geometrical features, to distinguish between healthy tissue, low grade and high grade cancer. Tutac et al. [8] initiated an innovative knowledge guided approach relying on the prior modeling of medical knowledge using ontology designed according to the clinical standard called Nottingham Grading System [9]. An extension to this work involving multi-scale approaches was proposed by Dalle et al. [10].

In close collaboration with a histopathology department, we built up a high-speed WSI analysis platform able to detect scale-dependent meaningful regions of interest in microscopic biopsy images. This platform is dedicated to the grading of breast cancer for prognosis purposes but the methodology we present here is quite generic. We use a standard optical microscope that can be found in most of the analysis laboratories in pathology or bacteri- 
ology (in our case, an optical microscope Olympus BX51, with 4X/10X/40X/60X/100X possible magnifications, Prior H101A ProScanII motorized X/Y stage and Z focus with a travel range of $114 \mathrm{~mm} \times 75 \mathrm{~mm}$ and a minimum step size of $0.02 \mu \mathrm{m}$, and a $1280 \times 1024$ pixels digital camera MediaCybernetics "EvolutionLC color" IEEE1394 MegaPixel). We use a MediaCybernetics controller connected to the microscope to perform an acquisition of high power fields/frames (in our study at 40X magnification according to the request of the pathologist for the high resolution analysis). The acquired 40X high power fields are stitched together in order to obtain the WSI.

To the best of our knowledge, most of the previous research works focused on the analysis of individual high resolution frames [11] and/or proposed solutions too computationally expensive to be applied at the WSI level [12]. A few notable exceptions [13] rely on the analysis of lower resolution images for the selection of regions of interest. Unfortunately, there is little correlation between low resolution images and the actual levels of nuclear pleomorphism observable at high resolution for instance. Therefore, even such methods proved to be inefficient for the particular issue of nuclear pleomorphism assessment on full biopsy slides. As a consequence, the time-efficiency problem posed by the extremely large scale of biopsy images (several thousands of frames) still lacks a practical solution.

In this work, we propose solutions to improve efficiency of such a microscopic platform both in terms of speed and precision, in particular with a multi-scale dynamic sampling approach and the use of GPU programming. The processing of a WSI starts by the detection of invasive regions of interest (ROI) at low resolution level (1.2X). This method relies on a bio-inspired visual information paradigm related to sparse coding and Graphics Processing Unit (GPU) implementation to dramatically speedup the processing line. This part will be detailed in Section 2. Once the ROIs are detected, a map of local cancer grades is established using a new generic multi-scale, computational geometry-based dynamic sampling method combined with high-resolution application specific image analysis algorithms. Then, this map is used to analyze the WSI within an operational time frame compatible with the pathology department's needs and on a par with the processing time of an experimented pathologist. This part will be detailed in Section 3. Finally, Section 4 elaborates on the results and validation issues and Section 5 is dedicated to conclusions, future research directions and challenges.

\section{Low Resolution Analysis and Sparse Coding}

Region of interest (ROI) detection is a fundamental phase of breast cancer grading for histopathological images. Pathologists identify ROIs to efficiently select the most important invasive areas for the grading process. Since neither pathologists nor computers are able to explore every details at high magnification within a reason- able time, the effective and efficient choice of the ROI is thus a critical step.

In this study, ROI detection is casted as as a classification problem. The low magnification analysis will determine if a given region is an invasive area in a similar manner as a pathologist would do when evaluating a biopsy. In order to mimic this behaviour, we exploit the relationship between human vision and neuroscience [14].

In the visual system, a set of opponent photo-receptors forms a Receptive Field (RF). These photoreceptors form a field which is called ganglion RF since they collect visual information and send neural spikes to a ganglion cell. Eventually, the ganglion cells produce various stimulations and send them to the primary visual cortex [15].

In the primary visual cortex, there are two major kinds of cells: simple- and complex-cells. Generally speaking, these cells produce two kinds of visual features: first- and second-order features [16]. The first-order feature contains the information of intensities of various color channels, and the second-order feature includes spatial variance of visual signal $[17,18]$.

In this study, we simulate some mechanisms of the human visual system, generate the first- and second- order features as the mechanisms in the human visual system. However, the human visual system and the brain is rather complex. Until now, it is not possible to simulate all of the function of the brain. Thus, in this study, we consider a classification algorithm for distinguishing the difference between the invasive area and normal tissue base on the visual information. Meanwhile, in order to accelerate this task, we make use of GPU technology. That is, the possibilities of the parallelization is also considered. As a result, we are able to use the proposing algorithm in practice.

\subsection{Parallel Computing with GPU}

A Graphics Processing Unit (GPU), has been involved in this study in order to achieve the goal of efficient performance. GPU is a specialized processor that offloads graphics rendering from the microprocessor. The highly parallel structure of the GPU makes the computing more effective than general-purpose CPUs for a range of complex algorithms. In a personal computer, a GPU can be present on a video card. The GPU were coined by NVIDIA marketing in 1999. Currently, most of the new desktop and notebook computers have integrated GPUs, which are usually far less powerful than those on a dedicated video card.

NVIDIA GPU comes with many streaming multiprocessors (SMs), each with many scalar cores (SPs) (see Fig. 1). Threads running on the cores within an SM can communicate with each other using shared memory. The unit of thread execution on the SP is a kernel and this is executed in concert with many other threads that form a block. Many blocks can be scheduled on the GPU, however a block is assigned only to one SM and as the results, threads across blocks should not rely on communications with each other. Blocks are scheduled by the GPU as and when the SMs have resources available to control them. 


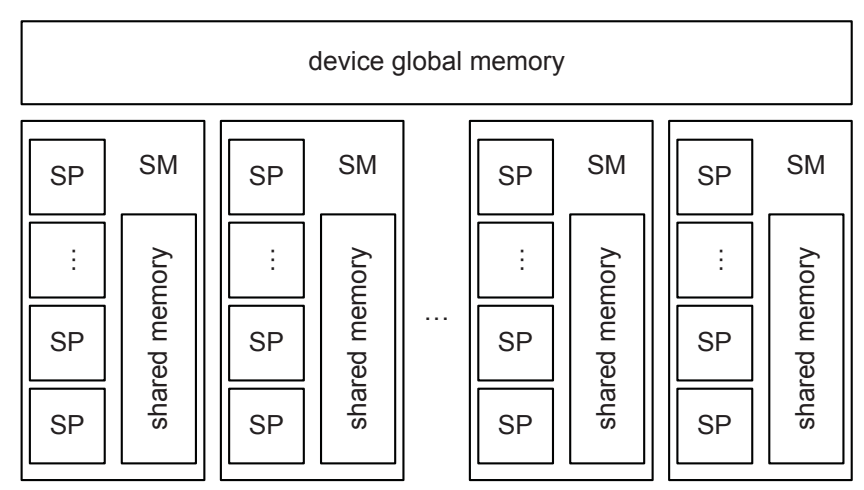

Figure 1: The architecture of GPU.
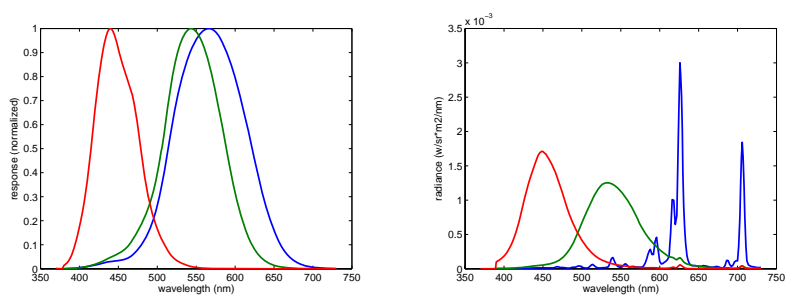

(a) Cone-cell sensitivities for var-(b) Spectrum of a typical CRT ious wavelengths of light. screen.

Figure 2: Retina and screen coefficients.

\subsubsection{Compute Unified Device Architecture}

Compute Unified Device Architecture (CUDA) is the computing engine in NVIDIA GPU that is accessible to software developers through industry standard programming languages.

CUDA Basic Linear Algebra Subprograms (CUBLAS) is an implementation of basic linear algebra subprograms on the top of the CUDA driver. It allows access to the computational resources of NVIDIA GPU. The library is self-contained at the API level. Thus, no direct interaction with the CUDA driver is necessary.

Although the CUDA and CUBLAS provide various advantages on the development. However, the algorithms in the proposed model is not that trivial since the limitations of the parallel processing architecture. For example, in the most recent version of CUDA, the version 3.0, some frequently used matrix operations, such as matrix inversion, is not included. Thus, for the sake of implementing the algorithms in the proposed model, some alternate mathematical matrix operations are required.

\subsection{Color Representation}

In this section, we aim at discovering the relationship between the contents on the screen displaying a breast biopsy image and the decision-making procedure which is performed when a pathologist is analyzing the image on the screen.

In order to extract the related visual reactions of human visual system, we need some parameters. First, the sensitivity of the photoreceptors in the visible spectrum. A well-known cone-cell sensitivity factor in visible spectrum is presented in Fig. 2a [19, 20]. Second, the radiance factor in the visible spectrum of the screen is required in order to evaluate the energy which might be received by the human visual system. Fig. $2 \mathrm{~b}$ shows a radiance factor in the visible spectrum of a typical CRT screen. Most modern screens provide Gamma correction, Gamma Display Functions (GDC) of the screen are also required to evaluate the radiance emitted by the screen based on the following transformation:

$$
\left[\begin{array}{c}
l \\
m \\
s
\end{array}\right]=a \cdot \mathbf{T} \cdot\left[\begin{array}{c}
\Gamma_{\text {red }}(r) \\
\Gamma_{\text {green }}(g) \\
\Gamma_{\text {blue }}(b)
\end{array}\right],
$$

where $r, g, b$ are the colors of the pixel, $\Gamma_{\text {red }}(\cdot), \Gamma_{\text {green }}(\cdot)$, and $\Gamma_{\text {blue }}(\cdot)$ are GDC functions used to reconstruct the Gamma correction, $s$ is a necessary scale in order to normalize the input values, $\mathbf{T}$ is a $3 \times 3$ matrix which is the linear combination of the cone-cell sensitivity factor and the radiance factor in visible spectrum. $l, m$, and $s$ represent the neural activities of various cone cells. Further details can be found in $[19,20,21]$.

There are many arguments that compare the pros and cons among various color spaces. According to Geusebroek et al., the opponent color theory can be applied to computer vision and implemented as the Gaussian color model $[22,23,24,25]$. In a Gaussian color model, the opponent colors can be obtained by:

$$
\begin{array}{r}
u_{\text {red }}=\log (l), \quad u_{\text {green }}=\log (m), \text { and } \\
u_{\text {blue }}=\log (s), \quad u_{\text {yellow }}=\log \left(\frac{1}{2}(l+m)\right) .
\end{array}
$$

Here $\left(u_{\text {red }}, u_{\text {green }}\right)$ and $\left(u_{\text {blue }}, u_{\text {yellow }}\right)$ are used to describe the opponent colors: Red-Green (RG), Blue-Yellow (BY), and Luminance $(\mathrm{L})$ :

$$
\left\{\begin{aligned}
v_{\mathrm{RG}} & =u_{\text {red }}-u_{\text {green }} \\
v_{\mathrm{BY}} & =u_{\text {blue }}-u_{\text {yellow }} \\
v_{\mathrm{L}} & =\frac{2}{3}\left(u_{\text {red }}+u_{\text {green }}+u_{\text {blue }}\right)-1
\end{aligned}\right.
$$

Those color pairs and the luminance information can be used to describe the visual signal of the human visual system $[15,22,21]$.

Based on these color information, the human visual system is able to extract such features from images. In our method, these feature extraction algorithms include intensity, color and texture perception.

\subsection{First Order Feature}

\subsubsection{Receptive Field}

In the human visual system, the Receptive Field (RF) of a ganglion is considered as the fundamental element [15]. In the first order extraction, the RF is defined as a set of pixels sampled over a specific area. Ideally, we should compute all of the pixels in this area. However, in order to reduce the computational cost, only some pixels in 


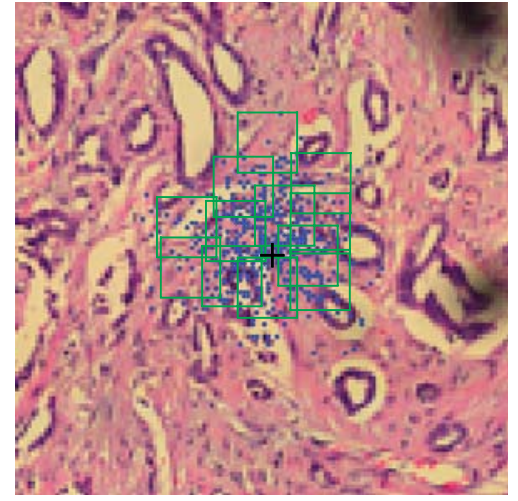

Figure 3: An example of breast biopsy (histopathology) image from our virtual microscopic platform. The resolution of the image is $8.3 \mu \mathrm{m} /$ pixel at $1.2 \times$ (12 times) magnification. One point (the black cross) is chosen by user click. For the first order feature, a number of points (the blue dots) are obtained based on multivariate Gaussian distribution. For the second order feature, a number of patches (the green rectangles) are obtained in a similar way. The covariances are $\Sigma_{11}=\Sigma_{22}=1000$ pixels and $\Sigma_{12}=\Sigma_{21}=0$.

this area are selected according to a sampling distribution. The sampling distribution $p_{\mathrm{s}}(\mathbf{l})$ is based on a multivariate Gaussian distribution such that

$$
p_{\mathrm{s}}(\mathbf{l}) \triangleq \mathcal{N}\left(\boldsymbol{\mu}_{\mathrm{s}}, \boldsymbol{\Sigma}_{\mathrm{s}}\right)
$$

where $\mathbf{l}=\left(l_{1} \ldots l_{i} \ldots l_{m}\right), l_{i} \in \mathbb{R}^{2}$ is a set of locations of data points, $\boldsymbol{\mu}_{\mathrm{s}}=[x, y]^{T}$ is the mean of the Gaussian distribution, $(x, y)$ is the location of the sampling point, and $\boldsymbol{\Sigma}_{\mathrm{s}}$ is the covariance matrix.

\subsubsection{First Order Feature Extraction}

When a RF is chosen, a set of pixel locations $\mathbf{l}=$ $\left(l_{1} \ldots l_{i} \ldots l_{m}\right), l_{i} \in \mathbb{R}^{2}$ is generated. We obtain the Hering's opponent color space: Red-Green (RG), Blue-Yellow (BY), and the Luminance (L) information as follows:

$$
v_{i}=\left[v_{\mathrm{RG}}\left(l_{i}\right), v_{\mathrm{BY}}\left(l_{i}\right), v_{\mathrm{L}}\left(l_{i}\right)\right], i=1 \ldots m .
$$

According to Geusebroek et al., the opponent color theory can be applied to computer vision and implemented as the Gaussian color model $[22,23,24,25]$. Thus, all $v_{i}$ can be used to generate a multivariate Gaussian distribution using a fast parallelized Expectation Maximization accelerated by GPU [26]:

$$
p_{1}(v) \triangleq \mathcal{N}\left(\boldsymbol{\mu}_{1}, \boldsymbol{\Sigma}_{1}\right) .
$$

The mean $\boldsymbol{\mu}_{1}$ and the covariance matrix $\boldsymbol{\Sigma}_{1}$, can be obtained. In our study, $\boldsymbol{\mu}_{1}$ and $\boldsymbol{\Sigma}_{1}$ are defined as the first order features.

\subsection{Second Order Feature}

Second-order features cannot be detected by mechanisms only sensitive to intensity changes. It is ubiquitous in visual scenes, but the neuronal mechanisms mediating perception of such stimuli are not well understood [27, 28].

Most research works agree that the second-order feature includes spatial variance of visual signal $[17,18]$. In order to extract the basis, various methods were proposed. Generally speaking, most of them invoke one or several basis analysing algorithms. Those algorithms include Gabor filtering, Principal Component Analysis (PCA), Independent Component Analysis (ICA), Sparse Coding, etc. Advantages and disadvantages of each of them have been actively compared. Olsausen et al. introduced the sparse coding for natural images in [29] assessing its performances in comparison with the PCA approaches (see Fig. 1 in [29]). They concluded that the principal components do not arise as a result of the oriented structures in whitened natural images. Karklin et al. in [30] and Willmore et al. in [31] compared more thoroughly various methods including sparse coding, Gabor filtering, PCA, and ICA in terms of cost and performance.

In this work, we propose a model based on sparse coding, similar to Karklin's model [32]. First, the basis vectors of various channels are extracted by sparse coding. Next, the related coefficients of these basis vectors for various images can be obtained. Third, the Gaussian distribution of these coefficients can be generated. Finally, the parameters $\mu$ and $\sigma$ are obtained and are considered as the second order features.

This model has the following advantages: it uses the same color space as the first order feature extraction. As a result, the computation time of the algorithm can be reduced. Another benefit is that some components in the system can be reused in this model. [33], Hyvärinen et al. indicated the similarity between the ICA and the sparse coding as well. Since the sparse coding has been proven suitable for implementation in GPU [34], this approach constitutes a definite, practical asset in order to increase the time-efficiency of the system.

The sparse coding is described as follows: assume an image of a natural scene contains $k$ pixels. Each image is stacked as a vector $\mathbf{x}_{i}$. Sparse coding $[29,35]$ suggests that $m$ natural scenes defined by $\mathbf{x}_{1} \ldots \mathbf{x}_{m} \in \mathbb{R}^{k}$ can be described in terms of a linear combination of $n$ basis vectors $\mathbf{b}_{1} \ldots \mathbf{b}_{n} \in \mathbb{R}^{k}$ and coefficients $\mathbf{s}_{1} \ldots \mathbf{s}_{m} \in \mathbb{R}^{n}$ with:

$$
\mathbf{x}_{i} \approx \sum_{j} \mathbf{b}_{j} s_{j, i}, \quad i=1 \ldots m,
$$

where $\mathbf{s}_{i}=\left[s_{1, i} \ldots s_{n, i}\right]$. The basis set can be over-complete $(n>k)$ if the maximum $k$ exists.

Basis vectors $\mathbf{b}_{1} \ldots \mathbf{b}_{n}$ and coefficients $\mathbf{s}_{1} \ldots \mathbf{s}_{n}$ are obtained using a training set $\mathbf{x}_{1} \ldots \mathbf{x}_{m}$. Thus, the optimization problem for $\mathbf{b}$ and $\mathbf{s}$ corresponds to the minimization 
of:

$$
\begin{aligned}
& \sum_{i=1}^{m} \frac{1}{2 \sigma^{2}}\left\|\mathbf{x}_{i}-\sum_{j=1}^{n} \mathbf{b}_{j} s_{j, i}\right\|^{2}+\beta \sum_{i=1}^{m} \sum_{j=1}^{n} \phi\left(s_{j, i}\right), \\
& \text { with }\left\|\mathbf{b}_{j}\right\|^{2} \leq c, j=1 \ldots n,
\end{aligned}
$$

where $\sigma^{2}$ is the variance of the noise, $\phi(\cdot)$ a sparsity function, and $\beta$ a constant.

Many methods can solve this optimization problem. A popular method is to consider one of the parameters in (8) as a convex function, and the other as the coefficient. By using an iteration loop, the minimization of (8) can be worked out. For example, first, hold the $\mathbf{b}$ and optimize the $s$. Then, hold the new $s$ and optimize the $\mathbf{b}$. The iteration continues until the cost function (8) is minimized. It can be implemented on GPU by the following algorithm proposed by Madhavan [34]:

- Parallelized coordinate descent algorithm: hold the $B$ in (8), and evaluate the $S$. Thus, (8) becomes the following minimization problem:

$$
\min _{x} f(x)=\frac{1}{2}\|y-A x\|^{2}+\gamma\|x\|_{1} .
$$

Thus, for each optimal $x_{j}$, the $x_{j}^{*}$ can be obtained by:

$$
x_{j}^{*}= \begin{cases}0, & \text { if }\left|-y^{T} a^{(j)}\right|<\gamma \\ \frac{y^{T} a^{(j)}-\gamma}{a^{(j) T} a^{(j)},}, & \text { if }\left(-y^{T} a^{(j)}\right)<-\gamma \\ \frac{y^{T} a^{(j)}+\gamma}{a^{(j) T} a^{(j)}}, & \text { if }\left(-y^{T} a^{(j)}\right)>\gamma\end{cases}
$$

- Learning bases using projected gradient descent: hold the $S$ in (8), and evaluate the $B$. Thus, (8) becomes the following minimization problem:

$$
\min _{x}\|X-B S\|_{F}^{2},
$$

The optimal $B$ can be obtained by projected gradient descent:

$$
B:=B-\eta \nabla_{B}\|X-B S\|_{F}^{2},
$$

constrained at each step by scaling $B$ down such that:

$$
\sum_{i=1}^{k} B_{i, j}^{2} \leq c, \forall j=1, \ldots, m
$$

where

$$
\nabla_{B}\|X-B S\|_{F}^{2}=-2(X-B S) S^{T} .
$$

\section{Receptive Field}

First, following (4), a set of data point locations $l_{1} \ldots l_{m} \in$ $\mathbb{R}^{2}$ are generated. Let $l_{i}$ be the center of an image patch, from which a set of patches $\mathbf{x}_{1} \ldots \mathbf{x}_{m}$ can be obtained:

$$
\mathbf{x}_{i}=\mathbf{I}\left(l_{i}-d\right),
$$

where $d=\left[n_{1}, n_{2}\right]^{T}$ and $\mathbf{I}$ represents a channel obtained from the input image, $-N \leq n_{1}<N$, and $-N \leq n_{2}<N$, and $N$ indicates the size of the patches. In the experiments, $N=10$ pixels. Since all patches $\mathbf{x}_{1} \ldots \mathbf{x}_{m}$ are captured from the same region, they share the same features. Thus, the texture can be encoded by coefficients $s_{j, i}$ in (7) and (8) with a set of basis vectors $\mathbf{b}_{j}$, which need to be obtained previously. All $s_{j, i}$ of RG: $s_{\mathrm{RG} j, i}$, BY: $s_{\mathrm{BY} j, i}$, and L: $s_{\mathrm{L} j, i}$ construct

$$
\begin{aligned}
& c_{j, i}=\left[s_{\mathrm{RG} j, i}\left(l_{i}\right), s_{\mathrm{BY} j, i}\left(l_{i}\right), s_{\mathrm{L} j, i}\left(l_{i}\right)\right], \text { and } \\
& i=1 \ldots m \text { and } j=1 \ldots n .
\end{aligned}
$$

All $c_{j, i}$ generate a multivariate Gaussian probability distribution using a fast parallel expectation maximization accelerated by GPU [26]:

$$
p_{2}(c) \triangleq \mathcal{N}\left(\boldsymbol{\mu}_{2}, \boldsymbol{\Sigma}_{2}\right) .
$$

Like the first order feature, the mean $\boldsymbol{\mu}_{2}$ and the covariance matrix $\boldsymbol{\Sigma}_{2}$ are obtained. In our study, $\boldsymbol{\mu}_{2}$ and $\boldsymbol{\Sigma}_{2}$ are defined as the second order features.

\subsection{Classification}

We described how to generate the first- and second- order features the same way as the mechanisms in the human visual system do it. For a pathologist, this kind of information is processed based on his/her knowledge, memory, and experience (the mental database). However, we simplify this issue and use a classification method to distinguish the invasive areas based on the visual information. Once again, we make use of GPU technology by leveraging the possibilities of parallelization to speed up again the analysis performance and provide a practical system for the end user in terms of time response.

The Support Vector Machine (SVM) is defined as following. A set of training data vectors $\mathbf{x}_{i} \in \mathcal{R}^{n}, i=1, \ldots, m$ is given. Each $\mathbf{x}_{i}$ includes the first and second order features $\boldsymbol{\mu}_{1}, \boldsymbol{\Sigma}_{1}, \boldsymbol{\mu}_{2}$, and $\boldsymbol{\Sigma}_{2}$. This set of data vectors is classified into two classes and is labeled by a vector $\mathbf{y}$ such that $y_{i} \in\{1,-1\}, i=1, \ldots, m$. Training a SVM as a classifier is equivalent to solve the following primal problem:

$$
\begin{aligned}
& \min _{\mathbf{w}, b, \xi} \frac{1}{2} \mathbf{w}^{T} \mathbf{w}+C \sum_{i=1}^{m} \xi_{i}, \\
& \text { subject to } y_{i}\left(\mathbf{w}^{T} \phi\left(\mathbf{x}_{i}\right)+b\right) \geq 1-\xi_{i},
\end{aligned}
$$

where $\xi_{i} \geq 0, i=1, \ldots, m$. The dual form of (18) is

$$
\begin{aligned}
& \min _{\alpha} \frac{1}{2} \alpha^{T} Q \alpha-\sum_{i} \alpha_{i}, \\
& \text { subject to } \mathbf{y}^{T} \alpha=0,
\end{aligned}
$$

where $0 \leq \alpha_{i} \leq C, i=1, \ldots, m, Q_{i j}=y_{i} y_{j} K\left(\mathbf{x}_{i}, \mathbf{x}_{j}\right)$, and $K\left(\mathbf{x}_{i}, \mathbf{x}_{j}\right) \equiv \phi\left(\mathbf{x}_{i}\right)^{T} \phi\left(\mathbf{x}_{j}\right)$. The SVM is implemented on GPU based on Carpenter's work [36]. In this method, $K\left(\mathbf{x}_{i}, \mathbf{x}_{j}\right)$ is defined as a Gaussian kernel:

$$
e^{-\lambda\left\|\mathbf{x}_{i}-\mathbf{x}_{j}\right\|^{2}}
$$




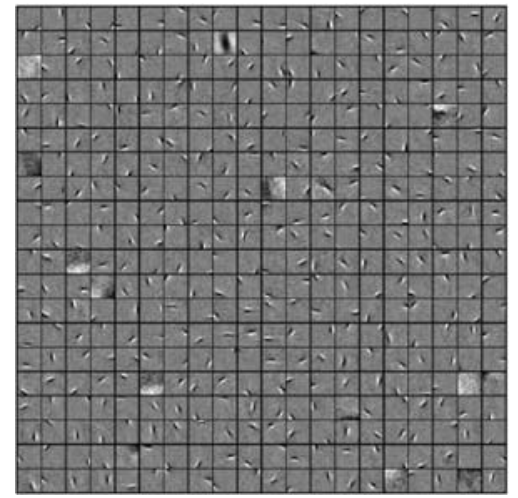

Figure 4: 400 basis vectors on $20 \times 20$ pixels image patches, extracted from digitized breast cancer biopsies (virtual microscope).

With a trained SVM, the hyper-plane is obtained as:

$$
\mathcal{H}(\mathbf{x})=\sum_{i=1}^{m} y_{i} \alpha_{i} \phi\left(\mathbf{x}_{i}\right)^{T} \phi(\mathbf{x})+b,
$$

and the classification is performed according to:

$$
\operatorname{sign}(\mathcal{H}(\mathbf{x})) \text {. }
$$

Note that $|\mathcal{H}(\mathbf{x})|$ is considered as the confidence degree of the classification.

\subsection{Training and Testing}

In the training phase, the pathologist provided digitized breast biopsies with annotated regions of invasive interest area. Then, the basis vectors for sparse coding were generated from these annotated samples. In the system, a GPU-based sparse coding was used in order to accelerate the process. We obtained 400 basis vectors on $20 \times 20$ pixels image patches, extracted from all channels (red-green, blue-yellow, and luminance) of the digitized breast cancer biopsies. The basis vectors of the luminance channel are presented in Fig. 4. Next, under the supervision of the pathologist, receptive fields (RFs) were selected by user click for both the first and second order features. These RFs included invasive and non-invasive areas validated by the pathologist (see Fig. 5a). The RFs are the training patterns of the GPU-based SVM [37].

In the testing phase, a set of RFs were selected on an input image based on a hexagonal arrangement (see Fig. 5b). The first and second order features of these RFs were classified by the trained GPU-based SVM [37] achieving an average 17 -fold speed up for the detection of invasive areas over the WSIs in comparison with a standard implementation. However, since only few regions were selected for classification in order to reduce computation time, an interpolation with a low-pass Gaussian filtering is applied to the SVM classification results (see Fig. 5c). The threshold for the invasive ROI detection is set to zero in Fig. 5d. Last, morphological opening and closing filters are applied on the results of the thresholding operation.

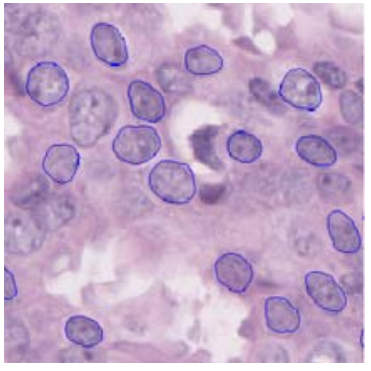

(a)

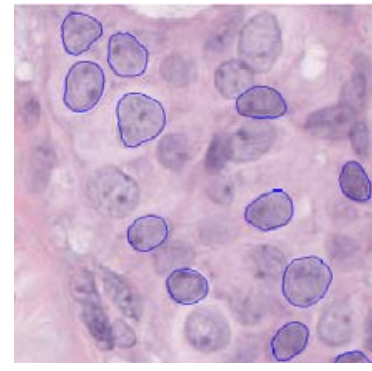

(b)
Figure 6: (a-b) Examples of segmented critical cell nuclei.

\section{Multi-scale Analysis and Sparse Sampling}

The Nottingham Grading System (NGS) [9] is based on an assessment of nuclear pleomorphism, mitotic counts and tubule formation. However, as for now the goal of the current study is not the clinical consistency but the time efficiency. Besides, for the grading assessment, we are on the process of building up a public image database with the corresponding clinical scores given by a group of expert pathologists. As for now, we trained the highresolution algorithm at the level of the frame on a semiconsistent image database of clinical cases that need to be consolidated in the near future. The global grading of the WSIs was given by one expert pathologist and still need to be confirmed by at least two other ones.

\subsection{Nuclear Pleomorphism Detection}

One important criterion of the NGS is the score of the nuclear pleomorphism which is assessed on segmented cell nuclei. Several methods have been proposed for the segmentation of cell nuclei in histological images $[6,38,39$, $40,41,42,43,44]$. These methods are based on traditional algorithms such as thresholding, watershed, morphological operations or active contours. The performance of these methods relies on the accurate segmentation of cell nuclei. This is computationally expensive when used to detect all the cells. In contrast, at least for nuclear pleomorphism scoring (one of the key component in NGS), according to the pathologist's knowledge and our experimental results, it is not necessary to segment all the cell nuclei but only critical cell nuclei affecting the score.

We refer the reader to [45] for more details about the high-resolution method that is based on cell segmentation using critical cell detection algorithm. It includes three distinctive stages: detection of the cell nuclei, followed by segmentation, and finally scoring of a population of cell nuclei (Fig. 6) at the frame level.

Then, the classification of the population of segmented cell nuclei is based on medical knowledge used by pathologists when scoring nuclear pleomorphism. Size, roundness and texture information of a population are extracted and used to build a Gaussian distribution that is compared to Gaussian models generated from training data 


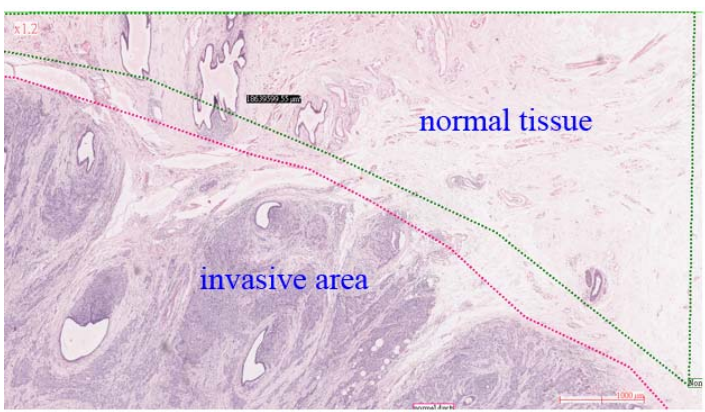

(a)

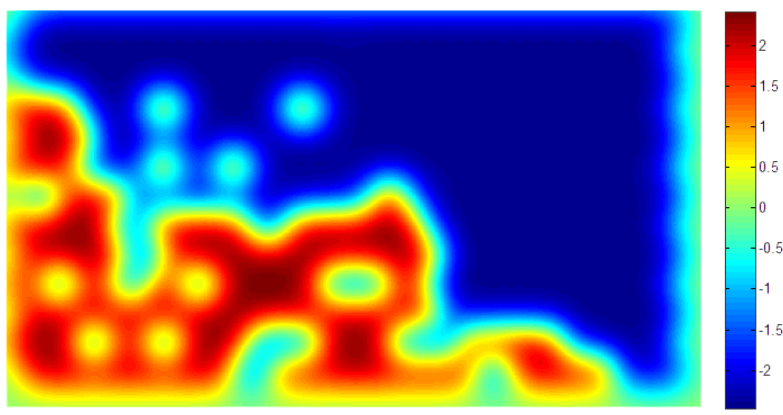

(c)

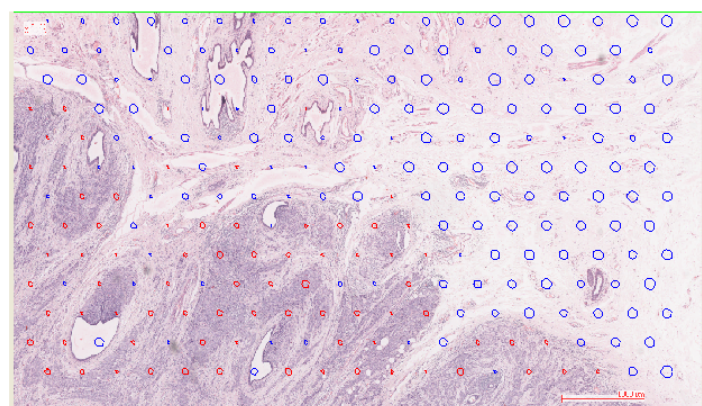

(b)

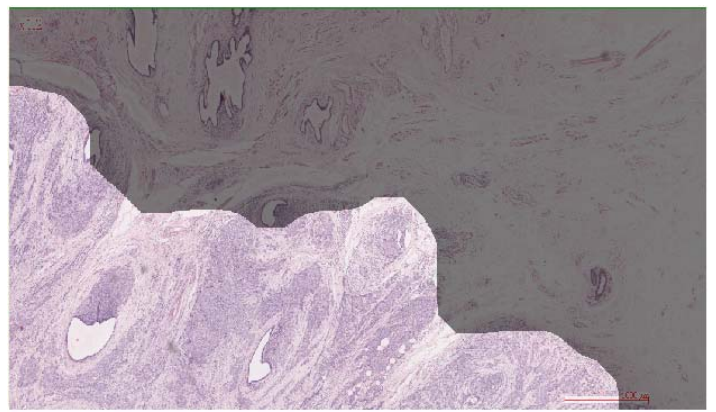

(d)

Figure 5: An example of ROI construction.

(a) Ground truth provided by pathologists over the input image. Usually the ground truth contains two regions: invasive and not invasive areas.

(b) Result of feature extraction and classification method on a set of equally distributed testing sites. The results are shown as circles. The red-circles indicate positive areas, and the blue-circles are negative areas. Note that the size of the circles is related to the hyper-plane $\mathcal{H}(\cdot)$.

(c) Low-pass filtering in order to estimate the characteristics on the areas between the testing points.

(d) The region of interest is obtained by thresholding.

for each score. The score of the population of cell nuclei is assessed by choosing the closest Gaussian Model to the Gaussian distribution representing the current population. Once again, the final clinical assessment of this score is still to be done. Only, assessment on a clinically semiconsistent database of frames has been performed so far. In particular, the final setting of the parameters weighting size, roundness and texture information for the computation of the local score still needs to be learned based on the database that is currently being built up for public release.

At the computer-aided diagnosis system level, the scoring of the nuclear pleomorphism can be used in two ways:

- after the ROI detection step(Section 2), to complete annotation of the frames included in the invasive area and build a systematic semantic indexing of those frames;

- as a component of the multi-scale WSI grading approach presented in following Section 3.2.

Since our frame-level, high-resolution grading algorithm, although selective, is still too slow to aggregate over one
WSI in order to give a global score in a realistic time response, we designed a generic multi-scale analysis method able to further accelerate the WSI grading in addition to the GPU-based ROI detection step. This corresponds more or less to the pathologist's way of processing the WSI by picking up about ten frames by a semi-informed cognitive sampling, but we do it here in a more systematic way using computational efficiency.

\subsection{Nuclear Pleomorphism Scoring using Multi-Scale Dy- namic Sampling}

The next step of the process requires the computation of a score for the nuclear pleomorphism over the selected ROIs and not only at the frame level. The score is computed out of the frame scores having the highest grade computed by our high-resolution nuclear pleomorphism scoring algorithm [45] applied over the whole ROI. However, a single ROI can be potentially very large, up to several thousands of high-resolution frames in some cases, making such exhaustive analysis intractable. Whereas the current research effort in histopathology image analysis focuses essentially on processing high-resolution frames and 


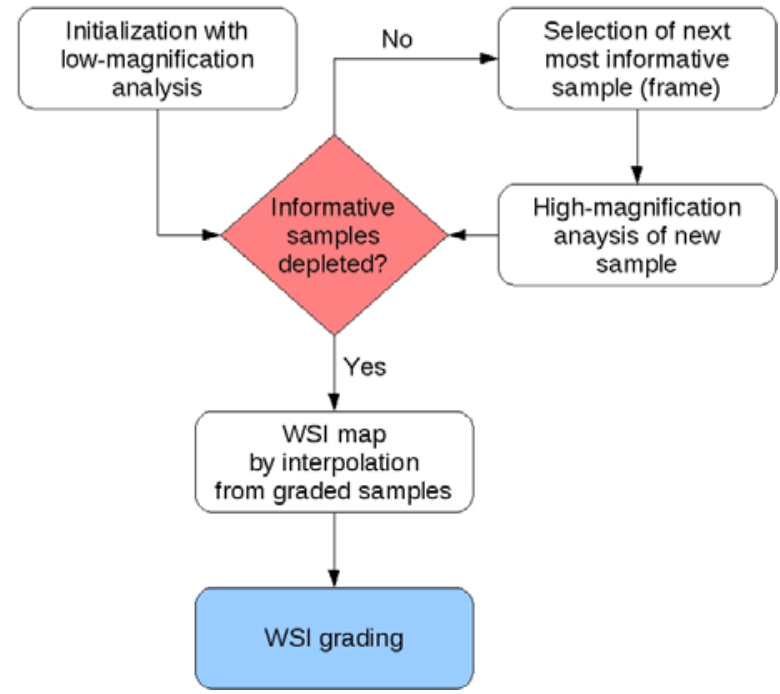

Figure 7: Multi-Scale Dynamic Sampling flowchart.

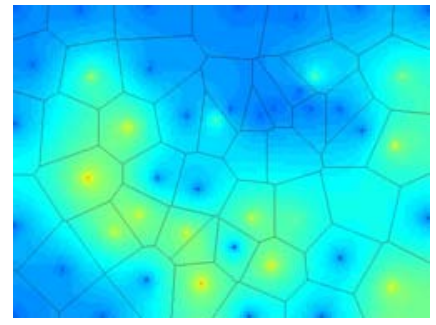

(a)

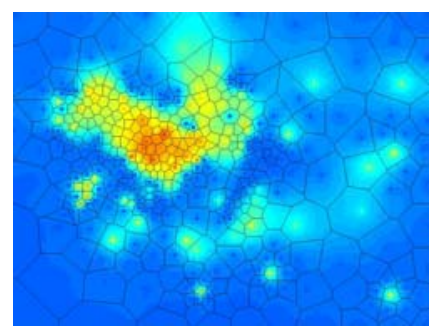

(c)

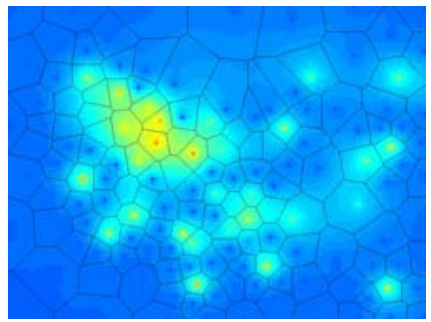

(b)

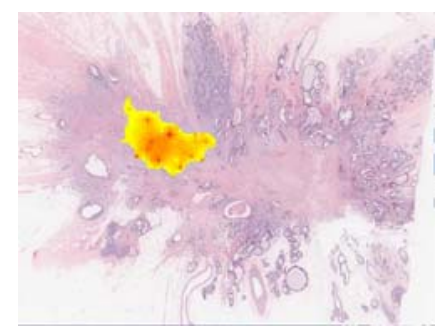

(d)
Figure 8: Dynamic sampling method applied over a WSI based on the ROI extraction. The incrementally constructed Voronoi diagrams are shown in black. Each cell contains a single sample at its center. The maps resulting from the interpolation are shown in colors. Hot colors represent higher pleomorphism values.

(a) After 50 samples: the whole ROI is being explored. No area seems favored.

(b) After 150 samples: the algorithm converges towards a high pleomorphism values area.

(c) After 400 samples: the sampling is very dense around this area and remains sparse in others.

(d) For illustration, the highest grading area obtained from the resulting map has been superimposed over a low resolution image of the ROI. Score for nuclear pleomorphism will be performed with frames picked from within this area. does not consider the problem at the WSI level, we make an attempt to develop an innovative method to rapidly identify the frames of interest necessary for the nuclear pleomorphism scoring.

Our algorithm aims at drawing a map of the nuclear pleomorphism levels encountered within the individual ROIs. In practice, the scoring is performed by the pathologist based on a few frames of interest picked up because potentially exhibiting the highest grade of cancer in the slide. Usually, the most cancerous areas can be identified with the regions having the highest degree of nuclear pleomorphism. It is the best indicator to obtain a global map of the cancer because it can be assessed locally (framewise) and is precisely quantifiable in a wide, continuous range (although the Nottingham grading system discretizes it). In comparison, mitoses are too sparse to be a good local indicator and tubular formations do not distinguish between different advanced cancers where tubules are absent.

A time-efficient exploration of every ROI is performed with an original technique that can be described as a multiscale dynamic sampling of the frames. The algorithm progressively identifies and focuses on regions showing a higher pleomorphism while discarding the rest. By avoiding an exhaustive analysis, it reduces the computation time by a factor 27 on average as shown by the experimental results in Section 4.

Let $I$ be a specific ROI from the WSI identified with the ROI selection algorithm described in Section 2. It comprises a large number of rectangular frames $x \in I$. For every frame $x$, the local nuclear pleomorhpism $S(x)$ (referred as "score") can be computed using the algorithm introduced in Section 3.1. The algorithm progressively identifies and focuses on interesting regions (with a high $S(x)$ ) while discarding the rest.

The following is a basic outline of this dynamic sampling algorithm (more details are available in [46]):

Step 1. First, the dynamic sampling algorithm is initialized using between 3 to 10 frames selected within the ROI. Then, the score $S(x)$ is computed for these initial samples. The selection is made using a simple low resolution analysis identifying the main histological components. This improves the robustness of the algorithm over an arbitrary selection of initial frames that is prone to miss entire components.

Step 2. Then, additional samples are dynamically selected and scored one-by-one in order to provide the most information at every step. The most informative samples are those which are both:

1. most distant from previous samples

2. within areas with a relatively higher pleomorphism

Computational geometry tools are used to efficiently identify samples verifying condition 1 . At each iteration, given $E$ the frames already sampled in the ROI $I$, we construct the Voronoi diagram of the centroids of the frames 
in $E$ denoted as $\operatorname{Vor}_{E}$. $\operatorname{Vor}_{E}$ is a collection of Voronoi cells $\left\{\nu_{x} \mid x \in E\right\}$, defined by $\nu_{x}=\{p \in I \mid \forall y \in I-$ $\{x\}, \operatorname{dist}(p, x) \leq \operatorname{dist}(p, y)\}$. The set of Voronoi vertices, later referred as $V_{E}$ are the vertices of the planar graph representation of $\operatorname{Vor}_{E}$. Voronoi vertices share the propriety to be locally the farthest position from their nearest neighbor in $E$, therefore in the case of our algorithm from already sampled frames.

Condition 2 is realized by selecting our next sample $x$ out of $V_{E}$ such that the score MaxScore $(x)$ of its highest scoring neighbor in $E$ is higher that $p \times \max _{E}$ where $\max _{E}$ is the currently observed maximal score among $E$ and $p \in$ $[0,1]$ is a preset parameter defining the selectivity of the algorithm.

In practice, we also impose that the distance between two samples must be larger than a theshold $d$ effectively defining the coarseness of the sampling. Both parameters $p$ and $d$ are dynamically adapted during the whole process to progressively increase the selectiveness and the fineness of the sampling. A pseudo-code for one iteration of the sampling algorithm is given in Algorithm 9.

Input: current samples $E, V o r_{E}, p, d, \max _{E}$

Output: updated values of $E, \operatorname{Vor}_{E}, \max _{E}$

1: compute $V_{E}$

2: sort $V_{E}$ according to decreasing distance to $E$

: for every $x \in V_{E}$ do

if $\operatorname{dist}(x, E) \geq d$ then

if $\operatorname{MaxScore}(x) \geq p \times \max _{E}$ then

$E=E \cup\{x\}$

update $\operatorname{Vor}_{E}$

$\max _{E}=\max \left(S(x), \max _{E}\right)$

beak loop

end if

else

beak loop

end if

end for

Figure 9: One iteration of the dynamic sampling algorithm

Step 3. Finally, the graded samples are used in order to interpolate the grade of all the frames in the ROI. The resulting map precisely details the regions showing the highest levels of nuclear pleomorphism which is sufficient to support an accurate global grading of the ROI. Frames of interest are selected from these regions and a scoring is performed with the algorithm described in Section 3.1.

Fig. 7 shows the proposed flowchart and Fig. 8 illustrates the algorithm output at different stages. It can be seen that the algorithm first has an exploratory behavior over the whole ROI, then converges towards areas with the highest pleomorphism, to finally refine the map around these areas.

The time complexity of our algorithm is equivalent to the cost of analyzing the sample frames. Indeed, as proved

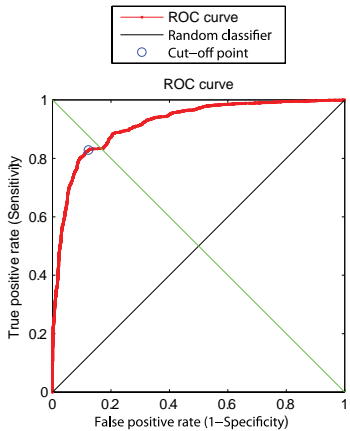

(a) The Receiver Operating Characteristic (ROC) curve.

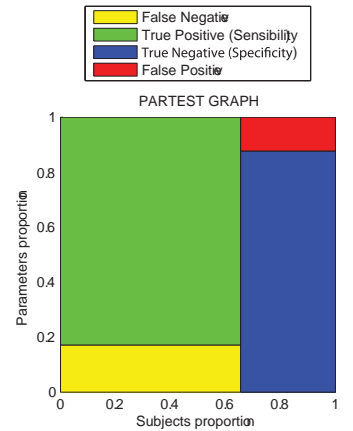

(b) The Clinical Test Performance.
Figure 10: Performance of our system. The AUC is 0.9186 .

by Ohya et al. in [47] the incremental construction of a Voronoi diagram has a constant average cost for every new added vertex (corresponding to our samples) and is negligible compared to the cost of analyzing a sample at high resolution.

\section{Results and Discussion}

Although application oriented, any microscopic WSI based system can leverage the ideas developed and assessed in this work to achieve interactive time applications. This section presents an experimental validation of the proposed time-efficient WSI analysis framework based on two major features of the breast cancer grading platform:

- the invasive area detection based on a GPU implementation and spare coding paradigm;

- the nuclear pleomorphism scoring map based on a sparse dynamic sampling algorithm.

The ground truth is provided by the pathology department from the National University Hospital, Singapore for nine breast cancer slides, with the annotation of the relevant invasive ROIs (the whole area covered by ROIs was outlined) and a global score $s \in\{1,2,3\}$ for the nuclear pleomorphism criterion. One slide is made up of about 4, 000 high power fields (representing the high resolution frames) of size $1024 \times 1024$ pixels, acquired at $40 \mathrm{X}$, with a resolution of $0.25 \mu \mathrm{m} /$ pixel, using Aperio ScanScope ${ }^{\circledR}$ CS fast scanner. This represents about 8 gigabytes of data per slide.

The microscope we presented in the introduction is used to design the high-resolution algorithm and to set up our own platform as well.But the ground truth must come from the digitized histopathological images acquired at the hospital. In practice, the pathologist is not able to analyze all the frames. Instead, he/she identifies the invasive areas at low resolution, before focusing on specific high resolution frames using his/her experience and his/her mental 


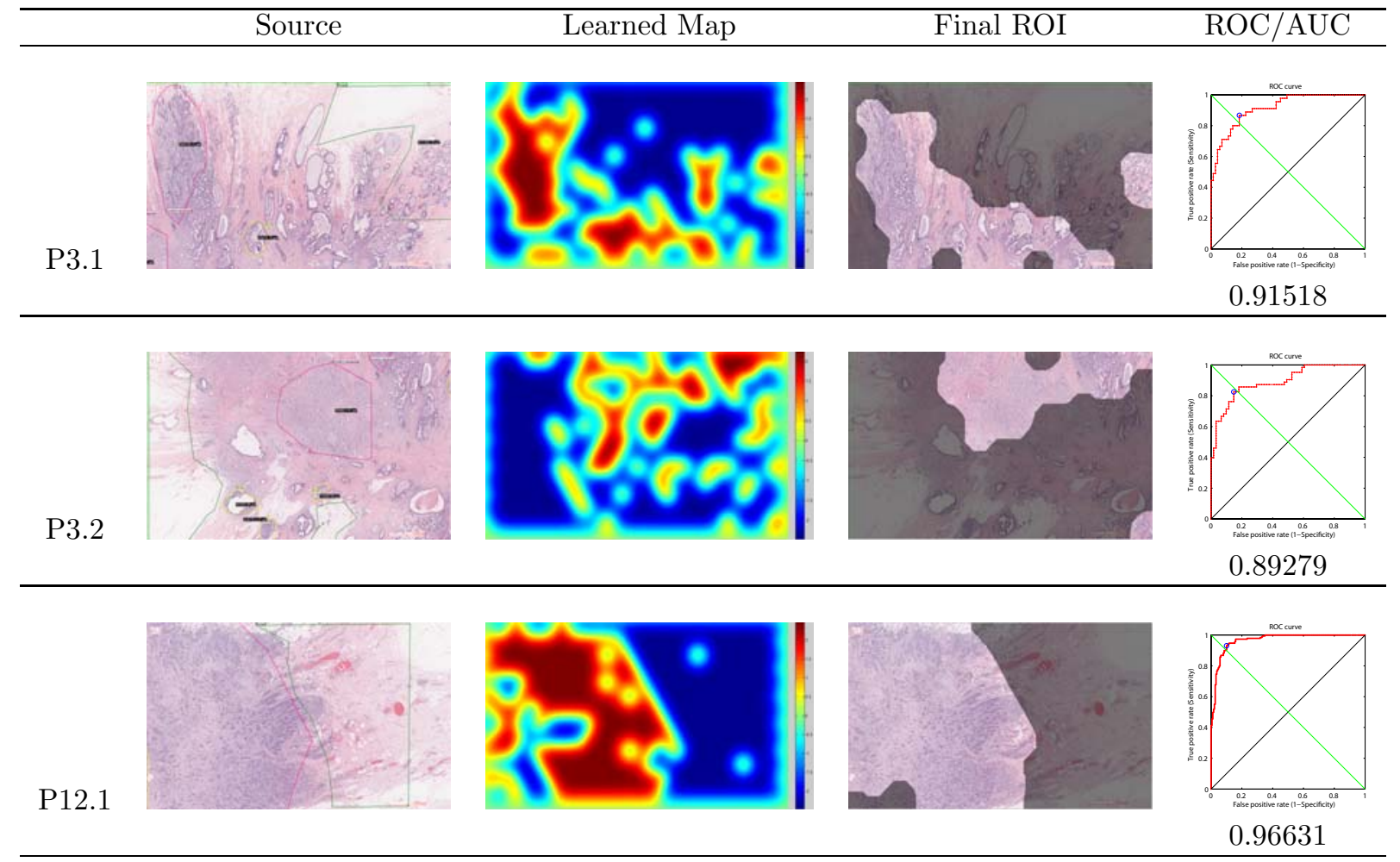

database. The grading is then performed based on these frames with, in a typical case, the examination of about only 10 to 40 high power fields.

The following results show that our system is able to provide a global score within an operational time frame of about ten minutes.

\subsection{Invasive area detection}

Fifteen ROIs were annotated out of nine digitized breast biopsy slides. Two of the ROIs are used as the training patterns. The global Receiver Operating Characteristic (ROC) curve and the Clinical Test Performance are shown in Fig. 10. The global Area Under the Curve (AUC) is 0.9186. We also computed the difference of ROIs between the results of ROI detection and the ground truth by the standard Jaccard index:

$$
c=\frac{R \cap G}{R \cup G},
$$

where $R$ is our result, and $G$ is the ground truth provided by the pathologist, and $c \in(0,1)$ is the covering rate. It should be noticed that the labeling of these ROIs by the pathologist was rather coarse and the delineation of these regions can be assessed as not accurate (see Table 1, left column). Anyway, due to the difficulty of such a task over a WSI we considered it as a first step towards a global assessment of our system. Despite this limitation, the average of all of the testing results is $c=0.7077$ which can be considered as a robust score according to the quality of the ground truth. Results ${ }^{1}$ are presented in Table 1 with from left to right the original slide, the first map issued by the learning process, the interpolated ROI to smooth the final result and the quantitative assessment by ROC curves.

In the design of our system, the results are determined by the sign of the hyper plane $\mathcal{H}(\cdot)$ which has been produced by the SVM-based classification. However, the test performance suggested that the optimal cut point be -1.1348 corresponding to the equal sensitivity/specificity cut-off point on the ROC curve. Although almost validated from a clinical point of view, the main objective of this study is to assess the methodology from a time-efficient point of view, which is actually the critical issue for WSI analysis.

In this perspective, the performance of GPU acceleration is presented in Table 2. The overall speed-up gain (sparse coding and SVM) is about 24-fold faster. The model of the GPU is GeForce $9400 \mathrm{M}$ from NVIDIA. The computer is Apple Macbook with Intel CPU Core 2 Duo with 4 gigabytes of memory. With a pre-trained kernel, the processing time allocated for the low resolution image ROI detection module is about 120 seconds a WSI. Once

\footnotetext{
${ }^{1}$ The full image set can be obtained from IPAL web site http: //ipal.i2r.a-star.edu.sg/project_MICO.htm
} 
Table 2: The performance of GPU acceleration.

\begin{tabular}{llll}
\hline & CPU & GPU & Speed-up Factor \\
\hline Sparse coding & 1406.759 & 56.297 & about $\times 25$ \\
SVM & 92.265 & 5.427 & about $\times 17$ \\
ECM & 26.314 & 2.58 & about $\times 10$ \\
\hline
\end{tabular}

these ROIs are detected in a reasonable time frame and at low resolution, a high-resolution but time-efficient analysis over these ROIs can be triggered by the dynamic sampling algorithm.

\subsection{Multi-Scale Dynamic Sampling}

In order to improve the precision of the sampling process, individual high resolution frames are split into four $512 \times 512$ pixel smaller frames. The map created by our algorithm is used to retrieve the frames corresponding to the highest pleomorphism scores. Therefore, we tested its performances for the retrieval of the set $R e l_{f}$ of frames in the ROI having a pleomorphism value of at least $0.9 \times \max$ where max is the global maximum pleomorphism value in the ROI. Ret $_{f}$ refers to the set of frames actually retrieved by our method for having an interpolated score of at least $0.9 \times$ max according to the map. We compute the precision and the recall defined by:

$$
\text { prec }=\frac{\left|\operatorname{Ret}_{f} \cap \operatorname{Rel}_{f}\right|}{\left|\operatorname{Ret}_{f}\right|} \quad r e c=\frac{\left|\operatorname{Ret}_{f} \cap \operatorname{Rel}_{f}\right|}{\left|\operatorname{Rel}_{f}\right|}
$$

For the sake of comparison, we also generate maps based on identical numbers of samples which were randomly selected. Comprehensive benchmark results for the nine slides can be found in Table 3 (the threshold value for the amount of selected samples is set to 500). Figures given in the case of random sampling are average values over 40 experiments. Note that the amount of "frames selected as samples" in Table 3 is the amount of frames selected by the dynamic sampling algorithm to create the map of the ROI and not the amount of high grade frames that are subsequently retrieved from the map.

Results show ideal overall performances of our algorithm with a consistent $100.0 \%$ precision, which means that frames selected by our algorithm are systematically representative of the highest pleomorphism values in the ROI. Nevertheless, we are conscious that these figures do not represent the real error risk in terms of precision due to the limited number of slides. As a matter of fact, unlike other medical imaging fields like mammography, in histopathology, no database is yet publicly available. In addition, fueled by the recent availability of instruments for digitizing microscope slides, the working group 26 of the Digital Imaging and Communications in Medicine $^{2}$
(DICOM) standard is about to release the supplement 145 "Whole Slide Microscopic Image IOD and SOP Classes". This explains that validation of systems dealing with WSI is still quite limited.

The recall rate fluctuates between $25.0 \%$ and $100.0 \%$ for an average value of $51.6 \%$ which is in any case sufficient to compute a robust nuclear pleomorphism on a large enough number of high grade frames. This means that the dynamic sampling method is a valid alternative to an exhaustive analysis in terms of quality of the scoring. These figures compare to the $45.8 \%$ precision and $4.0 \%$ recall averages obtained with a map generated from random sampling.

We also achieved a significant overall speedup: the proportion of analyzed frames ranges from $0.8 \%$ to $9.0 \%$ with an average of $3.3 \%$ of the ROI. Therefore, our method produced a clinically semi-consistent nuclear pleomorphisnm scoring requiring only $3.3 \%$ (on average) of the time required for an exhaustive analysis of the whole slide. As for now, a more dedicated local scoring must be designed framewise in order to achieve clinical consistency. However, the global time response will not be dramatically affected due to the generecity of the method in which any sophisticated local scoring function can be plugged in.

\subsection{Overall speed-up performance}

Processing the whole set of WSI high power fields would require several dozens of hours. For example, in [12], a multiscale stochastic texture-based algorithm classifies specific biological elements for virtual slide analysis of ovarian carcinoma cases in 100 to 300 hours on one processor of a 3 gigahertz Xeon quadriprocessor. This is prohibitive for clinical daily use ( but also for large scale biological modelling as needed for the Virtual Human Physiology efoorts currently carried out worldwide). Usually, a pathologist analyzes a breast biopsy slide in about ten minutes.

We implemented several mechanisms to speed-up WSI automatic processing and, regardless the accuracy performance, time required by our system to score a slide is on a par with pathologists' analysis time. In any case, improving accuracy performance should not degrade significantly the processing time performance achieved so far. The key idea is to mimic the pathologist's routine when he has to grade a slide. First of all, the most important point is to reduce dramatically the number of high power fields to be processed in detail. To reach this goal, in a first step, we perform ROI detection at low resolution (by using GPU, we achieve a speed-up factor of about 24-fold). In a second step, a multi-scale dynamic sampling selects a small number of high power fields with the highest concentration of pleomorphic cells. Precise cell detection and feature computation for scoring the slide, the most time consuming part of the scoring, is applied only to this small number of selected relevant high power fields. Only $3.3 \%$ of the high power fields of a slide are thoroughly analyzed on average.

\footnotetext{
${ }^{2}$ http://medical.nema.org/
} 
Table 3: Experimental results.

\begin{tabular}{|c|c|c|c|c|c|c|c|}
\hline \multirow{2}{*}{ Case id. } & \multirow{2}{*}{ Amount of frames } & \multirow{2}{*}{ Selected as samples } & \multirow{2}{*}{ Analyzed fraction } & \multicolumn{2}{|c|}{ Dynamic sampling } & \multicolumn{2}{|c|}{ Random sampling } \\
\hline & & & & precision & recall rate & precision & recall rate \\
\hline $\mathrm{P} 1$ & 8400 & 313 & $3.7 \%$ & 1.000 & 0.684 & 0.540 & 0.036 \\
\hline P2 & 2288 & 118 & $5.2 \%$ & 1.000 & 0.400 & 0.340 & 0.076 \\
\hline P3 & 9804 & 422 & $4.3 \%$ & 1.000 & 0.857 & 0.720 & 0.051 \\
\hline $\mathrm{P} 4$ & 3744 & 88 & $2.4 \%$ & 1.000 & 0.250 & 0.080 & 0.025 \\
\hline P5 & 5544 & 500 & $9.0 \%$ & 1.000 & 0.384 & 1.000 & 0.091 \\
\hline $\mathrm{P} 6$ & 22752 & 500 & $2.2 \%$ & 1.000 & 0.546 & 0.300 & 0.016 \\
\hline P7 & 14628 & 500 & $3.4 \%$ & 1.000 & 0.268 & 0.960 & 0.038 \\
\hline $\mathrm{P} 8$ & 14112 & 275 & $1.9 \%$ & 1.000 & 0.250 & 0.160 & 0.023 \\
\hline P9 & 10148 & 79 & $0.8 \%$ & 1.000 & 1.000 & 0.020 & 0.004 \\
\hline
\end{tabular}

\section{Conclusion}

From now, it is a great challenge to propose automated, time-efficient microscopic WSI analysis systems. The digital pathology world experiences the same overwhelming quality and quantity of data to be processed as the satellite world does. Not only has the system to be clinically pertinent, but it also has to provide results within reasonable time frame. This work has focused on the latter issue so far. However, to some extent, it has been proven clinically compliant. The Nottingham Grading System has not yet been fully implemented and only nuclear pleomorphism was tested. We proposed two solutions to speed up the overall time response of the system: the sparse coding paradigm embedded in a GPU framework for the preattentive part and the dynamic sampling algorithm to implement the informed picking up of frames operated by the pathologists. The assessment of our pleomorphism scoring showed room for improvement of our system from a clinical accuracy point of view but the overall architecture of the system has proven to yield preliminary clinical insights within a time frame on a par with the pathologists' one. As the nuclear pleomorphism scoring will get more accurate, the global clinical consistency of our system will improve without undermining much the overall time-efficiency of the proposed architecture within which a histopathological WSI is automatically analyzed in about ten minutes . All in all, this architecture can be applied to any microscopic WSI analysis systems regarding the multi-scale and GPU approaches and then tailored to every specific clinical or biological applications at the high-resolution level.

\section{Acknowledgment}

This study has been partially supported by the MMed$\mathrm{Web}^{3}$ grant A*STAR SERC 0521010103 (NUS R-252000-319-305) and by the ONCO-MEDIA ${ }^{4}$. We would like

\footnotetext{
${ }^{3}$ MMedWeb A*STAR/SERC: http://ipal.i2r.a-star.edu.sg/ projects.htm

${ }^{4}$ ONCO-MEDIA, ICT Asia programme: http://www . onco-media.com
}

to thank Dr. Jacques Klossa from TRIBVN company, Paris, France ${ }^{5}$ for his valuable expertise and support during this study. We would like to thank Mr. Xiong Wei from $\mathrm{A} * \mathrm{STAR} / \mathrm{I}^{2} \mathrm{R}$ for his contribution during the initial stage of the project. We also would like to thank Dr. Karklin Yan from New York University for the fruitful discussions about sparse coding and its implementation.

\section{References}

[1] T. Ming, The challenges and pitfalls of Biomedical imaging Perspectives of a Pathologist, in: SFBI'06, First SingaporeanFrench Biomedical Imaging Workshop, 2006.

[2] J. Estévez, S. Alayón, L. Moreno, R. Aguilar, J. Sigut, Cytological breast fine needle aspirate images analysis with a genetic fuzzy finite state machine, in: Proceedings of the 15th IEEE Symposium on Computer-Based Medical Systems, 21-26, 2002.

[3] F. Schnorrenberg, C. Pattichis, K. Kyriacou, V. M. Schizas, C. Schizas, Computer-aided classification of breast cancer nuclei, Technology \& Health Care 4 (1996) 147-161.

[4] S. Petushi, C. Katsinis, C.and Coward, F. Garcia, A. Tozeren, Automated identification of microstructures on histology slides, in: Proceedings of the IEEE International Symposium on Biomedical Imaging: Nano to Macro, vol. 1, 424-427, 2004.

[5] S. Petushi, F. U. Garcia, M. M. Haber, C. Katsinis, A. Tozeren, Large-scale computations on histology images reveal gradedifferentiating parameters for breast cancer, BioMed Central Medical Imaging 6 (2006) 1-14.

[6] S. Doyle, M. Hwang, K. Shah, A. Madabhushi, M. Feldman, J. Tomaszeweski, Automated grading of prostate cancer using architectural and textural image features, in: Proceedings of the 5th IEEE International Symposium on Biomedical Imaging: From Nano to Macro, 1284-1287, 2007.

[7] S. Doyle, S. Agner, A. Madabhushi, M. Feldman, J. Tomaszewski, Automated grading of breast cancer histopathology using spectral clustering with textural and architectural image features, in: Proceedings of the 5th IEEE International Symposium on Biomedical Imaging: From Nano to Macro, 496-499, 2008.

[8] A. E. Tutac, D. Racoceanu, T. Putti, W. Xiong, W. K. Leow, V. Cretu, Knowledge-Guided Semantic Indexing of Breast Cancer Histopathology Images, in: Y. Peng, Y. Zhang (Eds.), Proceedings of BioMedical Engineering and Informatics: New Development and the Future, vol. 2, 107-112, 2008.

[9] F. A. Tavassoli, P. Devilee (Eds.), World Health Organization Classification of Tumours, Pathology \& Genetics, Tumours of

\footnotetext{
${ }^{5}$ TRIBVN: http://www.tribvn.com/
} 
the Breast and Female Genital Organs, IARC Press, International Agency for Research on Cancer, 2003.

[10] J.-R. Dalle, W.-K. Leow, D. Racoceanu, A. E. Tutac, T. C. Putti, Automatic breast cancer grading of histopathological images, in: Proceedings of the 30th International Conference of the IEEE Engineering in Medicine and Biology Society, Vancouver, BC, Canada, 3052-3055, 2008.

[11] V. Ta, O. Lezoray, A. Elmoataz, S. Schupp, Graph-based Tools for Microscopic Cellular Image Segmentation, Pattern Recognition 42 (6) (2009) 1113-1125, special Issue on Digital Image Processing and Pattern Recognition Techniques for the Detection of Cancer.

[12] N. Signolle, B. Plancoulaine, P. Herlin, M. Revenu, TextureBased Multiscale Segmentation: Application to Stromal Compartment Characterization on Ovarian Carcinoma Virtual Slides, in: Proceedings of the International Conference on Image and Signal Processing, 173-182, 2008.

[13] M. Oger, P. Belhomme, J. Klossa, J.-J. Michels, A. Elmoataz, Automated region of interest retrieval and classification using spectral analysis, in: New trends in digital pathology: Proceedings of the 9th European Congress on Telepathology and 3rd International Congress on Virtual Microscopy, vol. 3, 2008.

[14] R. Miikkulainen, J. A. Bednar, Y. Choe, J. Sirosh, Computational Maps in the Visual Cortex, Springer, Berlin, 2005.

[15] D. H. Hubel, Eye, brain, and vision, W. H. Freeman, 1988

[16] G. Rhodes, Looking at faces: First-order and second-order features as determinants of facial appearance, Perception 17 (1) (1988) 43-63.

[17] I. A. Shevelev, Second-order features extraction in the cat visual cortex: Selective and invariant sensitivity of neurons to the shape and orientation of crosses and corners, Biosystems 48 (1) (1998) 195-204.

[18] C. C. Pack, B. R. Conway, R. T. Born, M. S. Livingstone, Spatiotemporal Structure of Nonlinear Subunits in Macaque Visual Cortex, The Journal of Neuroscience 26 (3) (2006) 893-907.

[19] CIE, An analytical model for describing the influence of lighting parameters upon visual performance - vol. 1, Tech. Rep., Technical Foundations, CIE 19/2.1, Technical Committee 3.1 , 1981.

[20] X. Zhang, S-CIELAB: A Spatial Extension to the CIE L*a*b* DeltaE Color Difference Metric, http://white.stanford.edu/ brian/scielab/, ????

[21] C. H. Huang, C. T. Lin, Bio-inspired computer fovea model based on hexagonal-type cellular neural network, IEEE Transactions on Circuits and Systems - I 54 (1) (2007) 35-47.

[22] J. M. Geusebroek, R. v. d. Boomgaard, A. W. M. Smeulders, H. Geerts, Color invariance, IEEE Transactions on Pattern Analysis and Machine Intelligence 23 (12) (2001) 1338-1350.

[23] J.-M. Geusebroek, R. v. d. Boomgaard, A. W. M. Smeulders, T. Gevers, Color constancy from physical principles, Pattern Recognition Letters: special issue on colour image processing and analysis 24 (11) (2003) 1653-1662.

[24] M. A. Hoang, J.-M. Geusebroek, A. W. M. Smeulders, Color texture measurement and segmentation, Signal Processing: special issue on content based image and video retrieval 85 (2) (2005) 265-275.

[25] H. Müller, N. Michoux, D. Bandon, A. Geissbuhler, A review of content-based image retrieval systems in medical applications - Clinical benefits and future directions, International Journal of Medical Informatics 73 (1) (2004) 1-23.

[26] N. S. L. P. Kumar, S. Satoor, I. Buck, Publisher, Fast Parallel Expectation Maximization for Gaussian Mixture Models on GPUs Using CUDA, in: Proceedings of the 11th IEEE International Conference on High Performance Computing and Communications, IEEE Computer, 103-109, 2009.

[27] J. Larsson, M. S. Landy, D. J. Heeger, Orientation-Selective Adaptation to First- and Second-Order Patterns in Human Visual Cortex, Journal of Neurophysiology 95 (2006) 862-881.

[28] H.-P. Frey, P. König, W. Einhäuser, The role of first- and second-order stimulus features for human overt attention, Perception \& Psychophysics 69 (2) (2007) 153-161.
[29] B. A. Olshausen, D. J. Field, Emergence of simple-cell receptive field properties by learning a sparse code for nature images, Nature 381 (13) (1996) 607-609.

[30] Y. Karklin, Hierarchical statistical models of computation in the visual cortex .

[31] B. Willmore, D. J. Tolhurst, Characterizing the sparseness of neural codes, Network: Computational Neural Systems 12 (2001) 255-270.

[32] Y. Karklin, M. S. Lewicki, Emergence of complex cell properties by learning to generalize in natural scenes, Nature 457 (2009) 83-86.

[33] A. Hyvärinen, P. O. Hoyer, A two-layer sparse coding model learns simple and complex cell receptive fields and topography from natural images, Vision Research 41 (18) (2001) 2413-2423.

[34] A. Madhavan, Using GPUs to speedup sparse coding algorithms applied to self-taught learning problems, Tech. Rep., Stanford University, 2008.

[35] H. Lee, A. Battle, R. Raina, A. Y. Ng, Efficient sparse coding algorithms, in: Natural Information Processing Systems Conference, 801-808, 2007.

[36] A. Carpenter, cuSVM: a CUDA implementation of support vector classification and regression, http://patternsonascreen.net/cuSVMDesc.pdf, 2009.

[37] B. C. Catanzaro, N. Sundaram, K. Keutzer, Fast Support Vector Machine Training and Classification on Graphics Processors, Tech. Rep., University of California at Berkeley, 2008.

[38] E. Cosatto, M. Miller, H. P. Graf, J. S. Meyer, Grading nuclear pleomorphism in histopathological micrographs, in: Proceedings of the International Conference on Pattern Recognition, 2008.

[39] Y. Hatanaka, K. Hashizume, Y. Kamihara, H. Itoh, H. Tsuda, R. Y. Osamura, Y. Tani, Quantitative immunohistochemical evaluation of HER2/neu expression with HercepTest in breast carcinoma by image analysis, Pathology International 51 (1) (2001) 33-36.

[40] A. S. Jadhav, P. Banarjee, K. Chaudhuri, J. Chatterjee, Quantitative analysis of histopathological features of precancerous lesion and condition using image processing techniques, in: Proceedings of the 19th IEEE International Symposium on Computer-Based Medical Systems, 231-236, 2006.

[41] H. Masmoudi, S. M. Hewitt, N. Petrick, K. J. Myers, M. A. Gavrielides, Automated quantitative assessment of HER-2/neu immunohistochemical expression in breast cancer, IEEE Transactions on Medical Imaging 28 (6) (2009) 916-925.

[42] K. A. Matkowskyj, D. Schonfeld, R. V. Benya, Quantitative Immunochemistry by Measuring Cumulative Signal Strength Using Commercially Available Software Photoshop and Matlab, Journal of Histochemistry \& Cytochemistry 48 (2) (2000) 303311.

[43] H. Soltanian-Zadeh, K. Jafari-Khouzani, Multiwavelet grading of prostate pathological images, IEEE Transactions on Biomedical Engineering 50 (2003) 697-704.

[44] W. Xiong, S. Ong, C. Kang, J. Lim, J. Lui, D. Racoceanu, K. Foong, Cell Clumping Quantification and Automatic Area Classification in Peripheral Blood Smear Images, in: The $2^{\text {nd }}$ International Conference on BioMedical Engineering and Informatics - BMEI'09, Tianjin, China, 2009.

[45] J.-R. Dalle, H. Li, C.-H. Huang, W.-K. Leow, D. Racoceanu, T. C. Putti, Nuclear Pleomorphism Scoring by Selective Cell Nuclei Detection, in: IEEE Workshop on Applications of Computer Vision, Snowbird, Utah, USA, 2009.

[46] A. Veillard, N. Loménie, D. Racoceanu, An Exploration Scheme for Large Images: Application to Breast Cancer Grading, Proceedings of the 20th International Conference on Patern Recognition .

[47] T. Ohya, M. Miri, K. Murota, Improvements of the incremental method for the Voronoi diagram with computational comparison of various algorithms, Journal of the Operational Research Society of Japan 27 (1984) 306-336. 
Chao-Hui Huang. He received his M.Sc. degree in computer science and information engineering from ChungHua University, Taiwan, in 2001, and His Ph.D. degree in electrical and control engineering from National ChiaoTung University, Taiwan, in 2007, respectively. He is currently a research fellow in Image \& Pervasive Access Lab (French National Research Center, CNRS UMI 2955) and School of Computing, National University of Singapore, (2008-2010). During his Ph.D. preparation, he was invited to serve as a visiting student scholar in University California, Berkeley, (2005-2006). He also has several years experience of project management, system analysis, and development in various world-class software and hardware companies, including International Business Machines (IBM), Taiwan-Hsinchu Office. Until 2010, he has published more than 20 technical papers in journals and conferences, and have been awarded a US patent and co-owned a copyright patent. The National Science Council, Taiwan, also has awarded him a potential young researcher scholarship in 2005. His current research interest is the bio-inspired computer vision and machine learning, including the fields of parallel computing, soft computing, computational neuroscience, visual psychology, neurobiology, and cognitive science, etc. He is also interesting on the software/hardware development for the bio-inspired computer vision and machine learning.

Antoine Veillard. . He received a M.Eng. degree from the Ecole Polytechique, Palaiseau, France in 2009 with a double major in computer science. He is currently pursuing his Ph.D. degree in computer science at the National University of Singapore in collaboration with IPAL (joint NUS/I2R/ASTAR/CNRS lab) in Singapore. His current research interests are about computer vision, computational geometry, computer algebra and machine learning in general.

Nicolas Loménie. He received his M.Eng. degree in Telecommunication from the French Institute for Telecommunications, Paris, in 1997, M.Sc. degree in Artificial Intelligence, Pattern recognition and Applications from the University of Paris 6, France the same year and Ph.D. degree in Computer Sciences from University Paris 5, France, in 2001. He is currently Associate Professor at University Paris Descartes and research fellow at IPAL (joint NUS/I2R/ASTAR/CNRS lab) in Singapore. His research interests are about image analysis, pattern recognition and computer vision in general. He has worked so far on object recognition issues in VHR satellite images, medical image screening and stereoscopic point set analysis and from a more theoretical point of view has developed original morphological mesh operators. He has been responsible for the image analysis and computer vision part of two ANR granted projects (REVES project - RIAM program and VISAVI project - RNTL program). For now, his current research topics include also knowledge-driven medical image analysis, in particular for the exploration of huge histopathological images.

Daniel Racoceanu. He received M.Eng. degree in Mechatronics from the Politehnica University of Timisoara in 1992, M.Sc. degree in Automatics and Industrial Informatics from the University of Technology of Belfort-Montbéliard (UTBM), France in 1993, Ph.D. degree in 1997 and HabilDr. degree in 2006 in Control and Computer Sciences from the University of Besançon, France. Daniel Racoceanu was Project Manager at General Electric Energy Products Europe, Belfort, France before joining, in 1999 a chair of Associate Professor at the Faculty of Sciences of the University of Besançon, France, where he coursed until 2005 in the field of artificial intelligence, computer vision, industrial informatics, automation, control science and production systems. Senior Scientist at the French National Research Center (CNRS), Daniel RACOCEANU is the Director (France) of Image \& Pervasive Access Lab (IPAL), the International Joint Research Unit (UMI CNRS 2955) created in Singapore between the CNRS, the National University of Singapore (NUS), the Institute for Infocomm Research (I2R) of the Singaporean Agency for Science, Technology and Research (A*STAR) and the University Joseph Fourier of Grenoble, France (UJF). From 1999 to 2005, Daniel Racoceanu was with FEMTO-ST/AS2M lab of Besançon, France, working in Diagnosis/Prognosis area using Artificial Intelligent Techniques (Dynamic Neural Networks; Fuzzy Logic, Neuro-Fuzzy Systems). Daniel Racoceanu's actual researches in IPAL focus on Intelligent Diagnosis/Prognosis Assistance using Medical Image and Knowledge-Based Reasoning Systems, Content and Context Based Medical Image Retrieval and Medical Multimedia Fusion.

Ludovic Roux. He received his Ph.D. degree in Computer Science from Paul Sabatier University, Toulouse, France in 1997. He has been a Research Fellow with Japan Aerospace Exploration Agency (JAXA), Tokyo, Japan, from 1997 to 1999, then a Software Engineer at Thales Communications, Massy, France. He is currently Research Fellow at IPAL (joint NUS/I2R/A*STAR/CNRS laboratory) in Singapore. His research interests are in image processing, soft computing and machine learning. 